\title{
On the prospects of using a phase transition in Ag nanoclusters for information recording processes
}

\author{
D. A. Bashkova, Y. Y. Gafner ${ }^{\dagger}$, S. L. Gafner \\ †ygafner@khsu.ru
}

Khakassian State University, 90 Lenin Av., Abakan, 655017, Russia

\begin{abstract}
To achieve a technological breakthrough in the creation of high-speed PCM cells, it is necessary to understand the nature of the switching effect of stable states and study the properties of the materials used for the working layer of a memory cell. The study of crystallization kinetics requires knowledge of the fundamental thermal properties of both crystal and amorphous states. If to consider all the chemical compounds tested for possible application in PCM memory, the metal nanoclusters of a relatively smaller size are of a special interest to analyze them for this task. The applicability of using individual nanoclusters of $\mathrm{Ag}$ as unites of storage of data bits in nonvolatile memory devices, the store capability of which is based on the principle of the phase change of the state of the carrier of information, has been estimated. Therefore, with the help of the molecular dynamics method on the basis of the TB-SMA potential, the simulation of melting-crystallization processes of Ag nanoparticles $(D \leq 10.0 \mathrm{~nm})$ has been performed. The influence of various conditions of crystallization on the formation of the internal structure in Ag nanoclusters is investigated. The limiting size of Ag nanoparticles is determined, for which a structural "orderdisorder" transition necessary for the data recording is still possible. It has been concluded that the best material for the memory cells, the store capability of which is based on the occurrence of phase transitions, is Ag nanoclusters with a diameter of $D \geq 8.0 \mathrm{~nm}$.
\end{abstract}

Keywords: molecular dynamics, silver, nanoclusters, phase transitions.

\section{Introduction}

Today, global leaders of the production of electronics actively develop the technology of a non-volatile phasechange memory (PCM), based on a phase transition from a crystalline state to an amorphous body [1-5]. The traditional types of memory used nowadays are based on the electrical charge storage. This makes the creation of smaller-sized structural elements technically difficult, as the contemporary semiconductor technologies have explicit quantum mechanical restrictions on the size of the used transistors. The conduction type (n-type or p-type) determines the minimum sizes comparable with a half the de Broil wavelength; for silicon, the values are 3.4 and 6.6 nanometers, accordingly. If the transistor sizes are reduced below these limit values, the electrons or holes will be actually "spread" across its space to not allow normal functioning of this device. Therefore, it is urgently necessary to develop the bases of nanoelectronics, operating according to absolutely new principles.

Besides, if compared with the flash-memory, which is the most extended today, the phase-change memory has a much higher information access rate, allows approximately 10 thousand more cycles of copying and can have higher density of information bit storage. That is why PCM can be considered as the most optimal option for the non-volatile memory to be extensively used in the next generation computer technologies. Due to its characteristics, PCM can replace the flash-memory, as well as the contemporary hard MD drives, dynamic and static random access memory and radiation-hardened memory of a special purpose. Nowadays such companies as Micron Technology, Intel, IBM, Samsung, Numonyx, etc. are involved in the industrial development of the phase-change memory technologies.

Currently, chalcogenides or GeSbTe alloy with a typical size of a memory cell of about 100 nanometers are applied as the PCM material $[6,7]$. Despite the small size of the cell, its heating requires rather high energy consumption - about $30 \mathrm{~mA} / \mathrm{Mbit}$, while the speed (crystallization time) being about 75 nanoseconds with an average overwriting resource of about $10^{12}$. The main technical problem consists in the difficulty of the active material recrystallization. Besides, the physical nature of the amorphous/crystal state transition is so far frequently an open issue. However, the evolution of the technique aimed at the creation of new non-volatile arrays of information requires different technical solutions [8-10]. First of all, it deals with the reduction of one bit storage area to several nanometers and reduction of the access time. All this requires a detailed research of the meltingcrystallization processes in such a small volume and in such a small time interval [11].

However, as all the potential materials found by us earlier $[12-14]$ for the use in PCM cells are quite expensive, we plan to carry out research aimed at finding materials of a lower price category. Based on the above stated, the main purpose of this work is a detailed study of the possibility to use clusters of silver as a single bit of information in long-term memory devices, based on the principle of changing the phase memory of the carrier. 


\section{The computer model}

It is well known that the technical application of clusters impose very serious requirements to the particle size, shape, internal structure and deficiency degree. The experimental detection of the mentioned values, especially for small clusters, faces great difficulties, despite the considerable development of the technology and a number of original techniques. As soon as the size of a particle becomes less than 4-5 nanometers (typical values used in catalysis and various biological applications), diffraction on X-rays ceases to show real data due to the technique features. In this case, it is possible to try electron microscopy or Mossbauer spectroscopy, but due to the frequent absence of adequate experimental results, many researchers use methods of computer simulation.

Today there are many simulation methods to study structural and thermodynamic properties of clusters. In our opinion, the most perspective method is the molecular dynamics (MD) method, which allows understanding the influence of various factors on different properties of nanoparticles at the atomic level.

To check the hypothesis of the use of single small nanoclusters of $\mathrm{Ag}(D \leq 10$ nanometers) as single bits of information, we will simulate the peculiar features of the structure formation in such clusters at different speed rates of thermal energy extraction, with the molecular dynamics method. After the analysis of different types of the potential energy representations for computing interatomic interaction power, we will mainly focus upon the use of the modified potentials of the TB-SMA interaction with the fixed truncation radius corresponding to the fifth coordination sphere inclusively [15]. It is evident that more complicated simulations similar to the ab-initio method are more realistic, but even the modern level of development of computer equipment makes it impossible to simulate clusters consisting of several hundred or thousand atoms with this method. On the other hand, the TB-SMA potentials were successfully applied in a number of cluster researches, and today they are one of the main potentials for simulation of metal nanoparticles.

Therefore, the simulation of heating will be carried out within the canonical ensemble based on interaction with the Nosé thermostat. The heating of each cluster begins with relaxation of the initial crystalline phase at the initial temperature, further the thermal energy supply continues till the final fracture of the long-range order in the nanoparticle. The structuring of small metal particles of the liquid phase will be examined with an Andersen's thermostat [16] that will allow one to simulate the cooling technique for nanoclusters with different rates. The simulated speed of cooling corresponds to the speed of the thermal energy extraction, which is technically already present in PCM devices. A cluster of each size should undergo a cycle of heating and cooling (thermocycling) ten times in order to determine the level of stability of the formation of the finite structure there.

The simulation temperature is defined with the average kinetic energy of atoms calculated on the basis of the Verlet high-speed algorithm [17] with a time step $h=1 \mathrm{fs}$. Computer program MDNTP developed by Dr. Ralf Meyer of Universität Duisburg (Germany), will be used for the analysis of structurization and thermal impact on clusters.

\section{Results and discussion}

In order to study the effect of kinetics on the formation of the structure of silver clusters, the process of melting of Ag nanoparticles with their subsequent cooling into the crystalline phase was imitated by the molecular-dynamics method. An analysis of the obtained results showed that when the diameter of silver nanoparticles exceeds $2.0 \mathrm{~nm}$, there is no spontaneous rearrangement of the cluster structure, i.e. the fcc structure of such clusters is thermally stable up to the melting point. For silver nanoparticles of a smaller size, the situation is much more complicated and numerous cases of thermally induced changes in the cluster structure have been observed, often occurring under different scenarios $[18,19]$. In accordance with the obtained data, it can be concluded that silver clusters with a diameter greater than $2.0 \mathrm{~nm}$ retain their original structure under the influence of the temperature factor and, from this point of view, they can be used as stable PCM memory cells.

At the next stage of the simulation, to determine the minimum size of the Ag cluster, which had a clear difference in the amorphous and crystalline structure at different rates of thermal energy removal, we used a group of clusters also having the same initial internal structure corresponding to an ideal fcc lattice, but with a diameter of $D=2.0-10.0 \mathrm{~nm}$. All clusters at $T=300 \mathrm{~K}$ underwent the mandatory procedure of thermal relaxation in order to optimize their internal structure and shape.

In the first stage, in the framework of the canonical NVT ensemble, when the number of particles $N$, the volume $V$, and temperature $T$ remain constant, using the Nosé thermostat, the simulated systems were heated stepwise with a step of $100 \mathrm{~K}$ to a temperature $T=1500 \mathrm{~K}$, much higher than their melting point, and at each fixed value they were maintained for $0.3 \mathrm{~ns}$.

In the second stage, using an Andersen's thermostat, the smooth cooling of silver clusters from the liquid phase to room temperature $T=300 \mathrm{~K}$ was simulated with some fixed velocities that correspond to the cooling time $\Delta t=0.1 ; 0.5$; $1.0 ; 2.0 \mathrm{~ns}$, without exposure to intermediate temperatures. At the same time, the simulated cooling rate corresponded to the rate of thermal energy removal, which is already used in PCM-memory devices, or somewhat exceeds it.

Thus, the dependence of the percentage ratio of the appearance of amorphous and crystalline structures on the cluster size and the cooling rate was investigated. An amorphous structure is understood here as a cluster with a strongly destroyed crystalline structure. The percentage probability of the realization of a particular structure means that $M$ individual clusters from the ensemble of $N$ clusters of the same diameter, considered in the course of modeling, are fully possessed by such a structure. The results of the simulation are presented in Table 1.

An analysis of the results obtained by cooling silver nanoclusters of different diameters from the molten state showed that there is a dependence of the crystalline 
formation process on the rate of heat energy removal. It can be seen that at a low heat dissipation rate corresponding to a cooling time of $2.0 \mathrm{~ns}$, all clusters have a crystalline structure. With a decrease in the cooling time to $0.1 \mathrm{~ns}$, a decrease in the percentage of occurrence of crystal structures is observed. However, at a high rate of thermal energy removal only for the cluster diameter $D \geq 8.0 \mathrm{~nm}$, all the clusters studied have an amorphous structure (Fig. 1).

Table 1. Probability of the appearance of crystalline and amorphous phases for Ag nanoclusters.

\begin{tabular}{|c|c|c|c|c|}
\hline \multirow{2}{*}{$\begin{array}{c}\text { Diameter of } \\
\text { the cluster } \\
D, \mathrm{~nm}\end{array}$} & \multirow{2}{*}{$\begin{array}{l}\text { Cooling } \\
\text { time } \\
\Delta t, \mathrm{~ns}\end{array}$} & \multirow{2}{*}{$\begin{array}{c}\text { Heat removal } \\
\quad \text { rate } \Delta T / \\
\Delta t \cdot 10^{12}, \mathrm{~K} / \mathrm{s}\end{array}$} & \multicolumn{2}{|c|}{$\begin{array}{l}\text { Probability of the } \\
\text { appearance of } \\
\text { structure phases, \% }\end{array}$} \\
\hline & & & Crystalline & Amorphous \\
\hline \multirow{4}{*}{2.0} & 0.1 & 12 & 30 & 70 \\
\hline & 0.5 & 2.4 & 70 & 30 \\
\hline & 1.0 & 1.2 & 90 & 10 \\
\hline & 2.0 & 0.6 & 100 & 0 \\
\hline \multirow{4}{*}{4.0} & 0.1 & 12 & 0 & 100 \\
\hline & 0.5 & 2.4 & 60 & 40 \\
\hline & 1.0 & 1.2 & 80 & 20 \\
\hline & 2.0 & 0.6 & 100 & 0 \\
\hline \multirow{4}{*}{6.0} & 0.1 & 12 & 15 & 85 \\
\hline & 0.5 & 2.4 & 10 & 90 \\
\hline & 1.0 & 1.2 & 80 & 20 \\
\hline & 2.0 & 0.6 & 100 & 0 \\
\hline \multirow{4}{*}{8.0} & 0.1 & 12 & 0 & 100 \\
\hline & 0.5 & 2.4 & 20 & 80 \\
\hline & 1.0 & 1.2 & 50 & 50 \\
\hline & 2.0 & 0.6 & 100 & 0 \\
\hline \multirow{4}{*}{10.0} & 0.1 & 12 & 0 & 100 \\
\hline & 0.5 & 2.4 & 0 & 100 \\
\hline & 1.0 & 1.2 & 30 & 70 \\
\hline & 2.0 & 0.6 & 100 & 0 \\
\hline
\end{tabular}

Therefore, based on the results of simulation of silver nanostructures, it can be concluded that its use as a memory element based on phase transitions is allowed, but with a cell size limitation exceeding $8.0 \mathrm{~nm}$.

\section{Conclusions}

To achieve a technological breakthrough in the creation of high-speed PCM cells, it is necessary to understand the nature of the switching effect of stable states and study the properties of the materials used for the working layer of a memory cell. Studying the crystallization kinetics requires knowledge of the fundamental thermal properties of both crystal and amorphous phases. If we consider all the chemical compounds tested for possible application in PCM memory, the metal nanoclusters of a relatively smaller size are of a special interest to analyze them for this task. Such an interest is connected with the relatively low cost of their synthesis and the rather a high probability to receive nanoparticles of the required size distribution.

In this work, the processes of the internal structure formation in Ag nanoclusters in conditions of ultrahighspeed hardening typical for PCM devices have been studied. To analyze the formation processes, we used the structureless clusters formed upon melting of primary fcc nanoparticles, and cooled them to room temperature. Various structural modifications were shown to form in cooling from the liquid phase, and some criteria of their stability were determined. In simulating, we revealed the role of the size factor and the heat removal rate in the structure formation in Ag clusters. It is necessary to take into consideration all these peculiarities when using small nanoclusters Ag in devices with phasechange memory of the carrier.

Acknowledgements. This work was supported by the Russian Foundation for Basic Research (project no. 18-42-190001 and 19-48-190002).
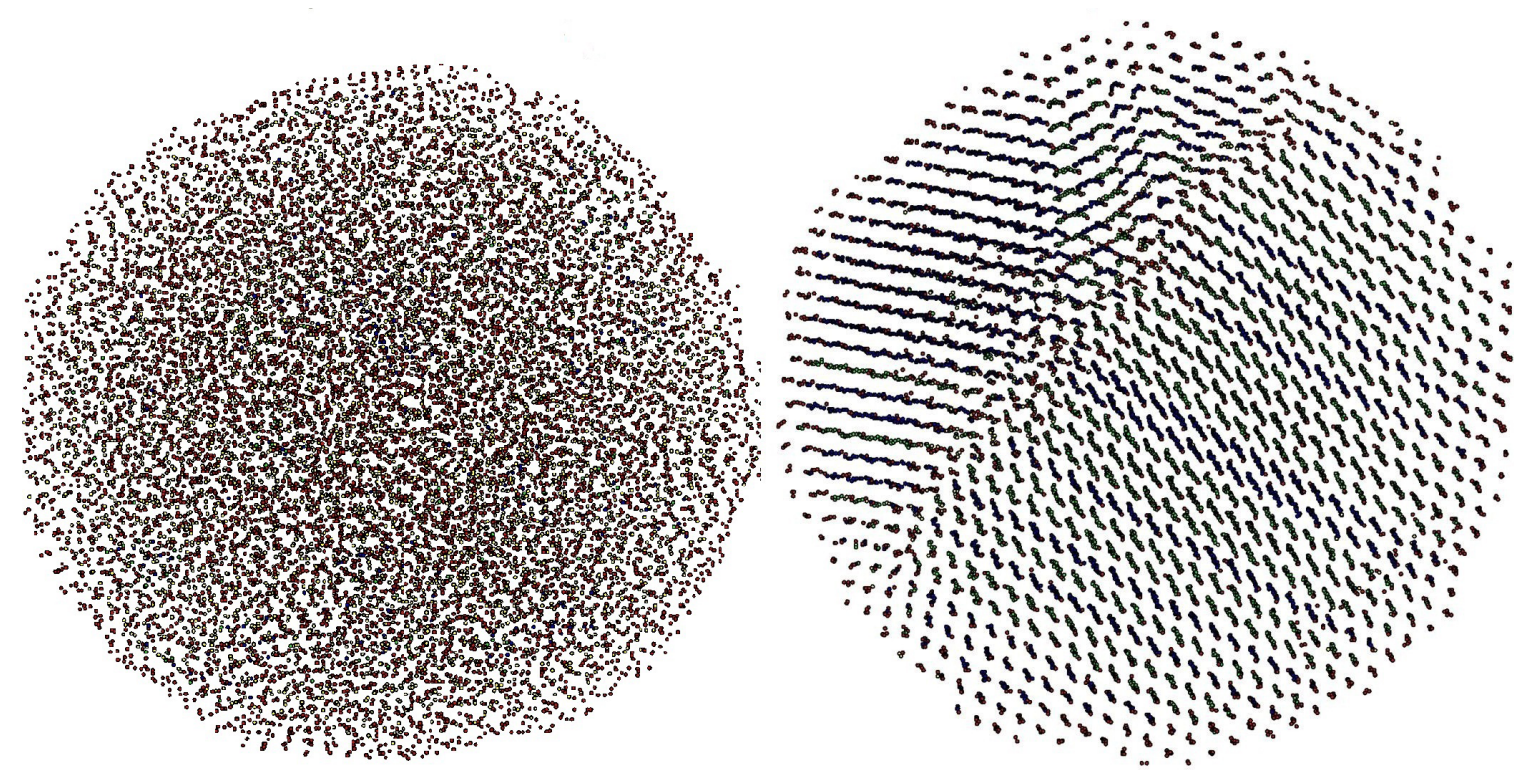

a

Fig. 1. Structural configurations of an Ag nanocluster with a diameter $D=8.0 \mathrm{~nm}$ with a different heat removal rate: amorphous (a); crystalline (b). 


\section{References}

1. G. W. Burr, M. J. Breitwisch, M. Franceschini, D. Garetto, K. Gopalakrishnan, B. Jackson, B. Kurdi. J. Vac. Sci. Technol. B. 28, 223 (2010). $\underline{\text { Crossref }}$

2. G. B. Beneventi. Characterization and modeling of phasechange memories. Lambert Academic Publishing (2012) $109 \mathrm{p}$.

3. M. Suri, O. Bichler, D. Querlioz, B. Traoré, O. Cueto, L. Perniola, V. Sousa, D. Vuillaume, Ch. Gamrat, B. DeSalvo. J. Appl. Phys. 112, 054904 (2012). Crossref

4. F. Wang. Non-Volatile memory devices based on chalcogenide materials. In: Flash Memories (ed. by I. Stievano). InTech Publ (2011) p. 197 -210. Crossref

5. Yu. I. Golovin. Introduction to Nanotechniques. Moscow, Mashinostroenie (2007) 496 p. (in Russian)

6. Y.C. Chen, C.T. Rettner, S. Raoux, G. W. Burr, S.H. Chen, R. M. Shelby, M. Salinga, et al. 2006 International Electron Devices Meeting. San Francisco, CA (2006) p. 777. Crossref

7. Z. Xu, B. Liu, Y. F. Chen, Z. H. Zhang, D. Gao. Solid-State Electronics. 116, 119 (2016). Crossref

8. D. Krebs, S. Raoux, C. T. Rettner, G. W. Burr, M. Salinga, M. Wuttig. Appl. Phys. Lett. 95, 082101 (2009). Crossref

9. D. Krebs, S. Raoux, C. T. Rettner, Y.C. Chen, G. W. Burr, M. Wuttig. Proceedings of the Materials Research Society. 1072, G06 (2008). Crossref
10. J.E. Brewer, M. Gill. Nonvolatile memory technologies with emphasis on Flash. A comprehensive guide to understanding and using NVM devices. New Jersey, A John Wiley \& Sons Inc. (2008) 759 p. Crossref

11. M. Salinga. Phase change materials for non-volatile electronic memories: Ph.D. thesis. RWTH-Aachen (2008) $224 \mathrm{p}$

12. Yu. Ya. Gafner, S. L. Gafner, L. V. Redel, Zh. V. Goloven'ko. Journal of Nanoscience and Nanotechnology. 14, 5138 (2014). Crossref

13. L. V. Redel, Yu. Ya. Gafner, S.L. Gafner, I.S. Zamulin, Zh. V. Goloven'ko. Physics of Metals and Metallography. 118, 452 (2017). Crossref

14. L. V. Redel, S.L. Gafner, Yu. Ya. Gafner, I.S. Zamulin, Zh.V. Goloven'ko. Physics of the Solid State. 59, 413 (2017). Crossref

15. F. Cleri, V. Rosato. Phys. Rev. B. 48, 22 (1993). Crossref

16. H. C. Andersen. J. Phys. Chem. 72, 2384 (1980). Crossref

17. T. Pang. An introduction to computational physics. University Press, Cambridge (2006) 385 p. Crossref

18. L. V. Redel, Yu. Ya. Gafner, S.L. Gafner. Physics of the Solid State. 57, 2117 (2015). Crossref

19. S. L. Gafner, D. A. Bashkova, Yu. Ya. Gafner. IOP Conf. Series: Materials Science and Engineering. 447, 012056 (2018). Crossref 